\title{
La Psiquiatría Forense, una disciplina dual en los Países Bajos
}

\author{
T.I. Oei MD PhD FCCP FRSM \\ Catedrático de Psiquiatría Forense \\ Tilburg University \\ P.O. Box 90153 \\ 5000 LE Tilburg \\ PAÍSES BAJOS
}

\begin{abstract}
RESUMEN - El autor realiza una defensa del papel tan importante que tiene reservada la Psiquiatría Forense en la Sociedad actual, de manera que ya representa una auténtica superespecialización a caballo entre la Medicina y el Derecho y con unas repercusiones éticas de primera magnitud en el campo del ejercicio de la Medicina. En el caso concreto de Holanda que posee una tradición reciente muy rica en este campo, el autor comenta diversos aspectos y entre ellos la necesidad de que se creen Cátedras de Psiquiatría Forense en las Facultades de Medicina para que los futuros médicos puedan beneficiarse de estas enseñanzas como ya lo hacen los estudiantes de Derecho que sí que disponen de Cátedras de Psiquiatría Forense en algunas de sus Facultades.
\end{abstract}

\section{Introducción}

La psiquiatría forense es una especialidad médica. Ofrece grandes posibilidades pero también tiene sus limitaciones lo cual ampliaré a medida que avance en este artículo. Del mismo modo, trataré de mostrar cuáles han sido los logros de la Psiquiatría Forense hasta ahora y establecer cuáles son sus objetivos para el futuro. Obviamente, he de ceñirme a los límites de un artículo.

Por ejemplo, estudiaremos los puntos en los que convergen el Derecho Penal, el Civil y el Administrativo, aunque el énfasis recaerá principalmente en cuestiones de Derecho Penal, una disciplina que también comparte algunas de sus bases con la ética médica. De hecho, no existe una Psiquiatría Forense que no tenga implicaciones éticas. No es, por tanto, una cuestión de "bueno/malo" sino de "humano/inhumano".

Hasta este punto, el Derecho Penal y la Psiquiatría Forense comparten las mismas bases; nada menos que encontrar el equilibrio entre los defensores de la Sociedad (por ejemplo, contra el peligro que suponen los pedófilos en 
las calles) por un lado, y por otro los que defienden el respeto por los derechos constitucionales de las personas. A ello podríamos denominarlo criterio "jurídico-ético".

Sin embargo, el campo de la Psiquiatría Forense continúa perteneciendo a la Medicina. Se suele pasar por alto este aspecto al observar que la Psiquiatría Forense tiene que actuar en los juzgados al mismo tiempo que se ve implicada en el proceso judicial. Cuando se trabaja dentro de las paredes de una Penitenciaría o en Instituciones de tipo judicial, el médico es el primero y desde luego la persona más importante encargada de atender a sus pacientes, los reclusos. La tensión que, inevitablemente surge entre la necesidad de informar, y el tratamiento debido a los detenidos, muestra claramente la necesidad que tiene el psiquiatra forense de tener una identidad específica como médico y de que exista un código de confidencialidad entre el médico y el paciente. Es necesario que las Facultades de Medicina presten más atención a la Psiquiatría Forense como disciplina básica. Saber si esto llegará alguna vez a ser posible, dependerá de los resultados que esta área sea capaz de producir. Mi tarea es la de continuar trabajando en este campo, utilizando todas las habilidades psiquiátricas que estén a mi alcance. La disciplina está tan estrechamente relacionada con la Medicina que no se puede limitar su existencia a las paredes de las Facultades de Derecho, además de que es primordial que se pueda facilitar la investigación y la práctica de esta Psiquiatría Forense en las Facultades de Medicina.

\section{La especialización}

La Psiquiatría Forense en los Países Bajos se desarrolló en el siglo XIX con el fin de que la información y el asesoramiento médico estuviesen a disposición de los jueces de los Tribunales de Justicia de lo Criminal, de los cuerpos legislativos (leyes civiles y administrativas), del Parlamento (legislaciones de tipo administrativo), de los abogados y sus clientes y de los acusados. Aumentaba la creencia entre la judicatura de que los médicos estaban cualificados para declarar en casos en los que los acusados, como resultado de sus trastornos mentales, eran incapaces de entender lo que habían hecho y por qué ello se consideraba como mal hecho. Tampoco podían prever las consecuencias de sus actos y, por esta razón, eran incapaces de enfrentarse a un juicio.

De manera automática, se consideró que los psicóticos no eran responsables de sus actos, al haber actuado cuando sus mentes estaban desequilibradas; en estos casos, los procedimientos legales fueron descartados y se procedió al ingreso incondicional en una institución psiquiátrica cerrada. Los cuerpos encargados de elaborar las leyes también llegaron a darse cuenta de que los enfermos mentales que habían sido detenidos y cuyo comportamiento no era propio de un psicótico en el momento en el que cometieron el delito, aún así no podían responder de sus actos y necesitaban seguir un tratamiento en lugar de ser encarcelados.

Fue en este momento cuando, a principios del siglo XX, se promulgó la Ley "A la discreción del Gobierno" (Conocida en el derecho británico como "Detention during her Majesty's pleasure") -que fue aprobada por la Segunda Cámara (la Cámara Baja) en 1925-. Finalmente, la ley entró en vigor en 1928 y esto significó que la práctica y el asesoramiento psiquiátrico se convirtieran en parte integrante de estos casos, terminaran o no en una Detención por Orden Judicial. En un principio, los vagabundos, mendigos y borrachos llenaban los Centros de Detección 
por Orden Judicial; les sucedieron personas que habían sido detenidas y padecían Trastornos de Personalidad, como los Psicópatas, llegando a convertirse en la población habitual de las Clínicas para Órdenes Judiciales en los años 40 y 50. A partir de 1970, incluso aquellos que fueron declarados culpables del robo de propiedades ajenas cumplían los requisitos necesarios para ser detenidos en instituciones por Orden Judicial. La ley fue rectificada en 1988, y a partir de este momento, cuando el juez consideraba la posibilidad de imponer una regulación mediante Orden judicial, era obligatorio consultar a dos expertos en comportamiento humano, uno de los cuales tenía que ser psiquiatra. De hecho, hasta 1997, la admisión en Instituciones Mentales por un periodo máximo de un año y las detenciones por orden judicial eran normales. Sin embargo, desde el mes de octubre del año pasado estas sentencias fueron desestimadas desde el punto de vista legal. La razón está en el hecho de que los psicóticos constituían una proporción sustancial (el 30\%) de las poblaciones de las Clínicas para Ordenes Judiciales, considerándose que las Instituciones psiquiátricas estaban mucho mejor equipadas para tratar a estas personas.

El juez siempre tiene la competencia legal para imponer un tiempo de detención por orden judicial, en tanto se pueda demostrar que el acusado padece algún tipo de trastorno psíquico en el momento en el que se produjo el delito o cuando se pronuncia la sentencia. La imposición de una Orden judicial en el caso de un sospechoso que no parece tener ningún trastorno psiquiátrico grave, es concebible (por ejemplo, cuando un experto examina a un sospechoso que no coopera y de quien se espera muy poco) pero es improbable, teniendo en cuenta que la detención por Orden Judicial se aplica principalmente con el fin de proteger a la
Sociedad del potencial criminal, altamente peligroso de un detenido que pueda presentar un trastorno mental. El tratamiento por Orden Judicial, a través del tratamiento obligatorio, también tiene como objetivo reducir el riesgo de una conducta reincidente, aunque ésta no sea una modalidad de tratamiento en sí misma.

Sin embargo, si la pérdida de la libertad es el objetivo principal de la sentencia, y si el detenido está tan gravemente trastornado que es imposible asegurar que era consciente de sus actos en el momento en el que cometió el delito, el juez tiene entonces la potestad de enviarlo a un hospital psiquiátrico general (sección 37 del Código Penal) por un periodo máximo de un año. Con la imposición de esta sanción se pretende que se lleve a cabo un tratamiento centrado en el paciente que en cuanto a los psicóticos se centra en la medicación anti-psicótica, ya sea provisional o definitiva, y a veces combinada con un periodo de tiempo en una celda de aislamiento.

Si, por otro lado, el delito (contra la propiedad más que contra las personas) no es tan grave o puede ser incluido dentro de la categoría de delitos especiales por los cuales es posible la detención por orden judicial, será entonces el juez quien tenga que decidir (en caso de que el trastorno que padezca el acusado requiriese tratamiento) si se le condena a pasar en la cárcel un periodo de tiempo que, en algunos casos, pudiera ser provisional. También puede optar, dentro del marco de las medidas excepcionales, por un periodo en prisión que sea corto y de carácter provisional siempre que el acusado acepte someterse al tratamiento. En el caso de haber cometido un delito por el que ha sido condenado a cuatro años de cárcel o más, el juez también puede considerar la posibilidad de imponer la sentencia de detención provisional por orden judicial, si un psiquiatra o 
un psicólogo llevan a cabo el tratamiento en un hospital psiquiátrico o en un Centro de Día Psiquiátrico. Este tipo de tratamiento (provisional) también se puede recibir en una Institución penitenciaria (por ejemplo, en el hospital de la prisión o en un lugar especial de la misma, el periodo correspondiente a la Orden Judicial no está incluido dentro del periodo de tratamiento, siendo ambos independientes), en una Institución del tipo de una Clínica para Ordenes Judiciales o en una Institución sanitaria (como el Centro Regional de Salud Mental o un Hospital General Psiquiátrico) o bien puede ser administrado de forma independiente por un psiquiatra o psicólogo.

De todas maneras son limitaciones implícitas en las regulaciones arriba mencionadas las siguientes:

1. La imposibilidad de condenar al acusado a prisión por más de tres años, incluso con una sentencia provisional (parcial), aunque su caso tenga características especiales, si se le ha declarado culpable de un delito grave (como los que atentan contra la vida humana).

2. La imposibilidad de considerar el tratamiento por Orden Judicial como tal, en caso de haber cometido un delito por el que no se puede imponer una condena de más de cuatro años de prisión. Comportamientos tan perturbados como el acoso, el chantaje y otros de este tipo pueden ser incluidos en el mismo grupo.

Los psiquiatras forenses judiciales también están obligados a prestar asistencia, según lo estipulado por el Ministerio de Justicia. La obligación de ofrecer una atención adecuada ha sido establecida con el fin de prevenir el deterioro de la condición del acusado o de la persona que dependa de la jurisdicción del Tribunal. En los últimos años también ha sido posible tratar a los presos, y un ejemplo de ello es que se ha puesto a disposición de los detenidos en espera de juicio una terapia previa que es llevada a cabo por los profesionales que pertenecen a Instituciones Psiquiátricas Penitenciarias.

Las limitaciones que tienen los tratamientos realizados bajo dependencia de la jurisdicción de los tribunales, incluyen:

1. La limitada posibilidad de aconsejar de un modo flexible una Orden Judicial o un tratamiento específico que de respuesta a las necesidades individuales, en caso de que la condena a prisión sea por un largo periodo de tiempo. La sección 13, junto con la sección 15 en el párrafo 5 de la Ley de Principios Penitenciarios, la sección 14 sobre la regulación penitenciaria (Admisión de los detenidos en una Clínica para Ordenes Judiciales debido a un trastorno psíquico o del comportamiento grave) se aplican en raras ocasiones, ya que son relativamente desconocidas, además del escaso interés que muestran los acusados ante la posibilidad de un tratamiento.

2. La casi total ausencia de autonomía por parte de los acusados a la hora de aceptar el desafío que supone el tratamiento, ya que normalmente ignoran todo lo relacionado con estos temas y, por tanto, no son conscientes de sus propias necesidades terapéuticas. Pero además al mismo tiempo, se sienten molestos cuando sus vidas privadas se convierten en objeto de estudio y dan lugar a conflictos personales y relacionales. El grado de tolerancia a la ansiedad, que a menudo sufre la influencia desfavorable del ambiente (ausencia de terapia social) y que predomina en el Departamento en cuestión, es normalmente el factor determinante para decidir si se incluye al acusado en un tratamiento de psicoterapia. 
3. Las limitadas posibilidades que existen para acceder a un tratamiento dentro del sistema de prisiones debido a la falta de psicólogos clínicos y psiquiatras.

4. La falta de presupuestos en el sistema de prisiones para pagar a un especialista en Comportamiento Humano. En 1996, la Comisión Balkema (Informe de la Comisión Noordsingel) recomendó que se aumentara el número de puestos tanto para el Departamento de Supervisión Individual como para los Departamentos de Cuidados Especiales.

\section{Logros académicos}

La Psiquiatría Forense es una disciplina académica relativamente joven. El primer Catedrático de Psiquiatría forense fue nombrado en Utrecht después de la Segunda Guerra Mundial, en el siglo pasado. La Cátedra de Psiquiatría Forense de Utrecht pertenecía a la Facultad de Derecho y fue ocupada por P.A.H. Baan, que era jurista y psiquiatra. Algunos aseguraban que Pieter Baan, afamado administrador y organizador, no fue aceptado en la Facultad de Medicina por su mentor y superior, el profesor Rümke y que por esa razón la Cátedra había sido concedida a la Facultad de Derecho. Por otro lado, hay que decir que Baan había terminado su formación práctica bajo la supervisión del Profesor Pompe, que en aquel momento era Catedrático de Derecho Criminal y amigo del profesor Kempe, el último profesor de Criminología. Juntos fundaron lo que se conoció más allá de las fronteras holandesas como la "Escuela de Utrecht" que se distinguía por ofrecer un enfoque optimista y amable al problema de los "psicópatas criminales" que se hallaban en las Instituciones Psiquiátricas Peniten- ciarias. Otro de los aspectos que les preocupaban era el del "encuentro" entre el juez y el acusado, ya que su objetivo era el de preparar al detenido para su rehabilitación dentro de la sociedad una vez hubiera salido de la cárcel. La "humanidad" fue el argumento utilizado para que el detenido pudiera recibir ayuda terapéutica y superar sus discapacidades sociales con el fin de que la resocialización y la reintegración no fueran sólo atractivos eslóganes sino también objetivos concretos. El nuevo plan también incluía formación complementaria y re-escolarización para que el desafío que supone la integración en la sociedad se convirtiese en una posibilidad real.

En la actualidad existen seis Cátedras de Psiquiatría Forense en los Países Bajos: En Utrecht y en Groningen (A.W.M. Mooij), en Leiden (A.M.H. van Leeuwen), en Nijmegen (H.J.C. van Marle), en Tilburg (T.I. Oei) y en Amsterdam (En su Universidad Libre, B.C.M. Raes). En Leiden y en Utrecht, las Cátedras de Psiquiatría Forense se clasificaron como "Ordinarias" y cada una estaba ocupado por un académico (C.M. van Esch, jurista, y F. Koenraadt, psicólogo, respectivamente) mientras que en otras Universidades se crearon Cátedras específicas para la Psiquiatría Forense.

A pesar de que todas las Universidades arriba mencionadas ofrecen básicamente el mismo tipo de programa académico, cada una tiene su estilo particular. Utrecht y Groningen, por ejemplo, prestan más atención a los aspectos fundamentales de la vida diaria (Mooij 2000), tales como el concepto de libertad, responsabilidad, culpabilidad, coacción e impulsión, además de aspectos empíricos (Utrecht) relacionados con la violencia en las relaciones, el tratamiento obligatorio y la multiculturalidad. Por otra parte, en Leiden, se presta más atención a aspectos relacionados con las pruebas judiciales 
cuando se investigan historias sospechosas (Janssen y Van Leeuwen 2000) de los individuos. En Nijmegen, se estudian en profundidad los Informes "Pro Justitia", aunque también juegan un papel importante los aspectos relacionados con la Medicina y los problemas relacionados con los aspectos forenses de la sexualidad (Van Marle 1999) En Tilburg, se centran en temas prácticos, y así estudian el papel que juegan tanto los hechos como la ficción en la investigación criminal, siempre y cuando mantengan relación con el proceso de estudio, con la obligación que tiene la Psiquiatría Forense de ofrecer la asistencia necesaria, y con la asistencia psiquiátrica relacionada con el Acta de los Alienados (Oei 2000). Y finalmente, en Amsterdam, una parte importante del programa académico está dedicada a tratar los aspectos fundamentales del tratamiento por Orden Judicial y a las cuestiones prácticas que rodean a estos casos (Raes 1999).

En las últimas décadas, también se han creado Cátedras de Psicología Forense (del Desarrollo) en la Universidad Libre de Amsterdam (R.A.R. Bullens, psicólogo del Desarrollo) y en la Universidad de Amsterdam (Ms. C. de Ruiter, psicólogo). Tanto la investigación como la práctica incluyen el cálculo del riesgo, la investigación en psicopatías, los estudios sobre la criminalidad en los adolescentes y la supervisión de los adolescentes en términos forenses (Oei y Groenhuijsen 2000).

De este modo, en los Países Bajos, se ha desarrollado una gran variedad de tipos de educación e investigación forense, sin que por ello esta disciplina haya perdido la posibilidad de modificar su campo de investigación. Dentro del amplio campo de la Psiquiatría Forense todos han podido encontrar su lugar y todas las iniciativas han contado con apoyo aunque las investigaciones objetivas que se han llevado a cabo han sido pocas.

\section{El terreno común en el que coinciden el Derecho Administrativo y el Civil}

El campo de la Psiquiatría Forense no queda limitado al ámbito de la Justicia Criminal o a los despachos de los jueces. Tanto la Justicia Civil como la Administrativa también recurren a la Psiquiatría Forense de vez en cuando. En el campo del Derecho Civil, del Administrativo y del Criminal, se producen consultas regulares (si no frecuentes) para saber cuál es el estado mental del detenido (¿cometió el delito siendo consciente de las consecuencias o sabiendo que es lo que ocurriría en cualquier caso?). ¿Existen pruebas suficientes que demuestren, por ejemplo, que el acusado era realmente consciente de la gravedad de la situación en la que se encontraba? ¿Se siente lo suficientemente libre el acusado como para afrontar la responsabilidad que supone haber tomado una decisión en un momento determinado de su vida? En el campo del Derecho Civil se puede cuestionar la capacidad del acusado para dar testimonio de forma voluntaria. La práctica de la Psiquiatría Forense también puede ser necesaria cuando se trata de ancianos, o de personas que padecen enfermedades psiquiátricas crónicas en el momento en que se cuestiona su capacidad mental y su habilidad individual para realizar operaciones tales como contratos (de compra y matrimoniales). Según el Derecho Eclesiástico, la incapacidad individual para mantener relaciones sexuales y para consumar el matrimonio (como resultado de un trastorno psíquico grave) puede ser considerada como razón admisible para el posterior divorcio y la anulación del matrimonio. Es necesaria una investigación más profunda para saber cómo se enfocan casos de este tipo de acuerdo con el Derecho Civil y la jurisprudencia necesaria. 
El psiquiatra forense también juega un papel importante cuando se pone en práctica la Ley de Indemnización a las Víctimas de Persecuciones ${ }^{1}$ (Visser 1997), y otras leyes similares. Este tipo de temas están relacionados con los aspectos mas básicos de la existencia humana y afectan a las alteraciones de la salud mental, la pregunta concreta sobre la culpabilidad del individuo ( $¿$ Era el acusado consciente de la gravedad de lo estaba haciendo? ¿Es capaz de distinguir lo que es correcto de lo incorrecto?) al igual que todo lo referente a las funciones cognitivas. Cuando, por ejemplo, una familia se divide (por ejemplo, cuando uno de los dos padres sufre una enfermedad mental y pretende modificar su testamento en contra del deseo de alguno de sus hijos) es necesario que el asunto sea examinado desde el punto de vista de la Psiquiatría Forense y, en interés de la objetividad, se ha de someter a las personas implicadas a una serie de pruebas neuropsicológicas. Este tipo de examen también es apropiado para valorar si la enfermedad requiere tratamiento y si un médico de la Sanidad Pública puede determinar hasta dónde llegar en lo que al tratamiento se refiere. La edad, la cronicidad, la calidad de vida, la falta de expectativas, el sufrimiento insoportable, el deseo personal de acabar con la vida mediante un suicidio realizado con la colaboración médica $y$, en resumen, muchos problemas fundamentales de este tipo necesitan de una orientación y criterios claros (Bannink et al. 2000).
Las cuestiones sobre Derecho Administrativo normalmente incluyen aspectos tales como el grado de capacidad que una persona tiene para trabajar, los intereses de los organismos gubernamentales, como por ejemplo los de los Ayuntamientos contra los intereses individuales e, igualmente los intereses de organizaciones privadas como las compañías de seguros y los Bancos contra los de una persona que ha sido acusada de un delito. Las preguntas relacionadas con la capacidad mental o psíquica de una persona para llevar a cabo tareas u operaciones militares también pueden entrar dentro de las competencias de un psiquiatra forense. En ocasiones, estos temas están relacionados con las reglas estatutarias del Código Highway según el Derecho Civil y el Administrativo (Oei 2000) ¿Hasta qué punto es el abuso en el consumo de alcohol y/o de drogas, el síntoma de que existe un trastorno de personalidad grave, de tal modo que el principio de la culpa in causa, no sea el asunto en cuestión a tratar? ¿Existe alguna prueba de que el acusado tuvo intención de cometer un acto delictivo o por el contrario no era consciente de lo que estaba haciendo (intento condicionado)?

Un campo importante para la Psiquiatría Forense es el de la legislación sobre la salud en sus diferentes aspectos. Y aquí, es corriente que las Leyes Penitenciarias coincidan. Por ejemplo, es en este campo cuando nos encontramos con los intereses legales del detenido, cuando es recluido por una orden judicial en

1. La Ley sobre Pensiones excepcionales 1940-1945. La Ley Indonesia sobre Pensiones excepcionales (Resistencia) 1940-1945. La Ley sobre Pensiones excepcionales de los marinos víctimas de la guerra 1940-1945. La Ley de Subsidios para las víctimas civiles de la guerra 1940-1945. La premisa de todas las leyes y regulaciones respecto a los damnificados física o psicológicamente como resultado de la Segunda Guerra Mundial intenta poder mantener el mismo estándar de vida que estas personas tenían antes de sufrir la invalidez que le llevó a perder su empleo. Ver W.D. Visser (ed.) Arbeidsongeschiktheid en traumatische (oorlogs)ervaringen (= Incapacidad para el trabajo y experiencias traumáticas de guerra). ICODO Foundation, Utrecht, 2nd edition, p. 19 onwards, 1997. 
instituciones estatales de forma temporal, de manera que los problemas que le afectaron indican que se le debe imponer un tratamiento. Queda claro, por supuesto, que la obligación del psiquiatra forense de proporcionar la asistencia adecuada exige al mismo tiempo que esté familiarizado con las posibilidades que ofrece la Ley en caso de urgencia (Berghmans y Widdershoven 2000).

Los conocimientos en Psiquiatría Forense también pueden ser necesarios en aspectos relacionados con el fraude y el crimen organizado. ¿Siguen siempre los estafadores un modelo definido, y por tanto predecible, o acaso en algunas ocasiones, el sujeto en cuestión padece un Trastorno de Personalidad grave de tipo narcisista, borderline, antisocial o psicopático?

Los conocimientos aportados por la Psiquiatría Forense en estas áreas, no pueden ni deben ser pasados por alto. Existen dos aspectos importantes en relación con ello:

1. Los juristas, miembros de la judicatura, (jueces, fiscales reales) y, si es necesario, los Servicios para la libertad condicional y la rehabilitación deben tener en cuenta la información y los consejos suministrados por la Psiquiatría Forense.

2. Los psiquiatras forenses judiciales pertenecientes al Servicio del Departamento de Justicia deberían publicar artículos sobre su trabajo de forma regular y por tanto, dar a conocer sus conocimientos a través de los medios. El papel público del psiquiatra forense le lleva a aceptar el hecho de que tiene que asumir responsabilidades en estos temas de vez en cuando. El código de comportamiento existente para los psiquiatras forenses que pertenecen al Departamento de Justicia es extremadamente explícito en ello, en el caso, por ejemplo de la contraprueba (Oei 1999), de los informes médicos, y de la conveniencia o no de una detención judicial (Oei 2000), etc.

\section{La Psiquiatría Forense y la Ética Médica}

El ámbito profesional de la Psiquiatría Forense cubre una parte importante del de la Ética Médica. No me limitaré aquí a la Ética Médica en particular a pesar de que algunos aspectos están directamente relacionados con el trabajo que ejerce el psiquiatra forense. Las cuestiones que rodean a la eutanasia, a la manipulación genética, y otros problemas similares tienen connotaciones tanto médico-éticas como psiquiátrico forenses. Las preguntas relacionadas con la Ética y la Política convergen igualmente en el campo de la Psiquiatría Forense, con aspectos que tienen que ver con la asistencia a inmigrantes ilegales y a su tratamiento médico-psiquiátrico $^{2}$ (Van Kalmthout 2000).

Los médicos que poseen buena voluntad se rigen por la ética médica y por ello surgen preguntas sobre la influencia que tiene el negocio y los aspectos comerciales sobre la práctica psiquiátrica y sobre la necesidad de que los médicos adopten una actitud independiente con respecto a la industria farmacéutica en su vida profesional, así como en cuanto a los programas de capacitación y de formación continuada (Oei 2000).

2. Comparar con A.M. van Kalmthout. De medisch-psychiatrische zorg van psychisch gestoorde illegalen: een juridische taxatie (= El cuidado médico psiquiátrico de los inmigrantes ilegales psíquicamente enfermos; una evaluación jurídica). En: T.I. Oei, M.S. Groenhuijsen (ed.), Forensische Psychiatrie anno 2000, Deventer: Gouda Quint 2000, p. 223-235. Y también: Top justicie: definitief geen tbs illegalen (= Top justicie: definitely no Court Order 
La Psiquiatría Forense de hoy en día es tan completa y multiforme que la formación en Psiquiatría Forense necesita de su específico programa educativo. Los psiquiatras no pueden justificar por más tiempo, desde el punto de vista de la Ética Médica, su visión de la disciplina como secundaria. El hecho de que existan psiquiatras forenses judiciales, significa en sí mismo, que ha llegado la hora de que esta área llegue a ser reconocida como una superespecialización de gran importancia. Se creó una Fundación Nacional en los Países Bajos con el propósito de establecer un programa de formación obligatorio para los especialistas en el Psiquiatría Forense que obtuvo críticas positivas.

Las actividades que se realizan dentro del campo de la Psiquiatría Forense son importantes y por ello se ha creado una asociación científica para promocionarlas. La Psicoterapia Forense es una disciplina independiente que no existe tan sólo para tratar con psicoterapia a los detenidos por orden judicial. El juez siempre será un factor determinante a la hora de programar los tratamientos como tales, teniendo en cuenta que es él quien determina cuánto tiempo determinado tratamiento puede darse bajo la jurisdicción propiamente judicial. No será la primera vez que desperdiciar la ocasión en este campo ha demostrado como puede ser autopunitiva y para ello sólo tenemos que contemplar los casos en los que el terapeuta se ha convertido en la víctima del detenido ${ }^{3}$.

\section{La Psiquiatría Forense y la investigación}

En las últimas décadas, la investigación en Psiquiatría Forense se está convirtiendo en algo cada vez más complejo. Todos los ejemplos de exámenes que se han llevado cabo y que han sido incluidos dentro de la Psiquiatría Forense son los siguientes: el grado de peligrosidad y riesgo de que vuelva a cometerse un acto delictivo -inherente a cualquier delito grave-, el grado de culpabilidad, la necesidad de determinar los hechos $\mathrm{y}$ actos valiosos para el forense especialista en comportamiento, la necesidad de llevar a cabo evaluaciones en el campo de la psicoterapia forense como las terapias cognitivas ofrezcan éstas o no perspectivas útiles, los examenes neurobiológicos de personas que padecen trastornos del comportamiento, los modelos de examen epidemiológico diseñados para avanzar en determinados temas clínicos, la valoración de la adicción clínica. De hecho, el examen neurobiológico de los trastornos del comportamiento agresivos,

illegal immigrants), de Volkskrant (periódico diario nacional), 22-11-00, p. 3, en el que el Ministerio de Justicia se espera que declare que sospecha que son responsables de sus acciones cuando en realidad no es así, o cuando el sentido de la responsabilidad esté disminuido; ello conlleva la imposición mediante sentencia, de prisión con el resultado de que los inmigrantes ilegales gravemente afectados en su salud mental terminan legalmente en prisión con la amenaza de poder ser deportados, lo cual es una situación contraria a los acuerdos internacionales. Ello significa que estos inmigrantes legales enfermos no reciban el tratamiento adecuado, lo que les viene a crear nuevas dificultades, debiendo tener en cuenta que su condición psíquica requiere una apropiada Orden Judicial o el tratamiento en un Hospital Psiquiátrico General.

3. En el caso de una intimidación agresiva o sexual el manejo de las manifestaciones transferenciales y antitransferenciales es muy difícil y la psicoterapia no puede conceder a nadie total inmunidad pero es como una vacunación que puede ayudarle. 
por ejemplo, ha adquirido grandes proporciones y los resultados tangibles están a punto de salir a la luz. Las preguntas sobre la causa y las consecuencias continúan siendo importantes aquí. ¿Es la experiencia de un acontecimiento importante en la vida un requisito o causa para adoptar una actitud delictiva (por ejemplo, manifestarse utilizando una agresión socialmente inaceptable)? Y si la respuesta es "sí", ¿cómo podemos categorizar este tipo de trastorno agresivo? ¿Qué tipo de acontecimiento vital, o evento imprevisto, realmente causó, o dio lugar, al desarrollo de un Trastorno de Estrés Post-traumático, por ejemplo? ¿Qué se puede obtener al categorizar las enfermedades y sus síntomas?, o por el contrario, ¿deberíamos concentrarnos más en el aspecto dimensional de los conjuntos de síntomas? Este tipo de preguntas están siendo planteadas en las Facultades de Medicina de todo el mundo y son de vital importancia para la existencia de una Psiquiatría Forense que se respete a sí misma. Además, como ciencia del comportamiento, su objetivo es el de seguir jugando un papel importante en el modo en el que la Justicia se lleva a la práctica y en el de averiguar cuál es la apreciación que se tiene desde fuera de dicha práctica (Tuinier 2000).

\section{En conclusión}

La Psiquiatría Forense es una disciplina fascinante que, en el ámbito de proporcionar información a la judicatura, además de atender a los enfermos psíquicos y a los desfavo- recidos de la Sociedad supone un desafío para la práctica médica. Todo gira en torno a los intereses de los individuos y las Instituciones, siendo su principio básico el de propugnar el respeto por uno mismo en equilibrio con el respeto hacia los demás, sin tener en cuenta si esto va a afectar a los intereses del juez, del gobierno, del acusado o del propio psiquiatra forense. El médico siempre tiene que respetar las normas y valores que prevalecen en la Sociedad que le rodea ${ }^{4}$.

Existe una necesidad de educación y de práctica en este campo especializado que se denomina Psiquiatría Forense. Sin embargo, por el momento, no existe una sola Facultad de Medicina en los Países Bajos que pueda presumir de tener (senior) profesores, o Catedráticos de Psiquiatría Forense. Aparentemente, la disciplina está considerada como la "cenicienta" académica de las ciencias médicas. Es ésta una actitud que, desafortunadamente, ha dejado un vacío en el campo de la educación e investigación de la Psiquiatría Forense y esto significa que los estudiantes de Medicina no llegan a conocer esta importante área. Si la Ética puede convertirse en un tema fundamental para los estudiantes de Medicina, por qué no así la Psiquiatría Forense. Los Países Bajos disfrutaron de un periodo -desde 1969 a 1986cuando el Catedrático Prof. W. Goudsmit, ocupó las Cátedras de Psiquiatría Forense, tanto en la Facultad de Medicina como en la de Derecho de la Universidad de Groningen. No hay duda de que ha llegado el momento en que el mismo tipo de doble ligazón vuelva a reinstaurarse. Después de todo, la Psiquiatría Forense existe gracias a

4. Si se demuestra que un dentista/doctor es un consumidor excesivo de alcohol, puede ser forzado a abandonar su práctica clínica. Ver Trouw (periódico diario nacional), 21-11-00, p. 4 que recoge el hecho de que en Groningen un dentista fue sometido al Tribunal Regional Disciplinario. 
las ciencias médicas y legales juntas y precisamente por ello es una disciplina dual. Tanto la práctica como la investigación han de construirse sobre bases médicas y jurídicas, ya que después de todo, la diosa Justicia y Aesculapius fueron aliados durante siglos. Ya es hora, por tanto, de que el Ministerio de Sanidad, Bienestar y Deportes en colaboración con el Ministerio de Justicia facilite, con un respaldo a la vez moral y financiero, la investigación y la práctica de la educación en Psiquiatría Forense en las Facultades de Medicina de los Países Bajos.

\section{Bibliografía}

BANNINK, M., VAN GOOL, A.R., VAN DER HEIDE, A., VAN DER MAAS, P.J. Psychiatric consultation and quality of decision making in euthanasia. The Lancet, 356, 2067-2068, 2000

BERGHMANS, R.L.P., WIDDERSHOVEN, G.A.M. Dwang en drang in de psychiatrie (Compulsion and impulsion in psychiatry). In: T.I. Oei and M.S. Groenhuijsen (eds.), Forensische Psychiatrie anno 2000, 77-94. Gouda Quint, Deventer. Compare further the responsibility of the tresting specialist in terms of medical care. L. Oates courts' role in decisions about medical tratment, British Medical Journal, 321, 1282-1284, 2000.

JANSSEN, S.L.J., VAN LEEUWEN, A.M.H. Wie van de Drie? Dissociatie in de forensisch psychiatrische rapportage (= Which of the three? Dissociation in forensic psychiatric reporting). In: T.I. Oei and M.S. Groenhuijsen (eds.), Forensische Psychiatrie anno 2000 (= Forensic Psychiatry anno 2000), 119-127, GoudaQuint, Deventer 2000.

MOOIJ, A. De fundamentele verantwoordelijkheid van de hulpverlener (= The fundamental responsibility of the care-giver). In: J. Graste and D. Bauduin (eds.), Waardenvol werk. Ethiek in de geestelijke gezondheidszorg (= Values in Work. Ethics in mental health care). Van Gorcum \& Comp. Ltd., 66-77, Assen 2000.

OEI, T.I. Een kijkje in de behandelkamer van de forensisch psychiater in de P.I. "De LIssel" (= A glimpse into the surgery of the forensic psychiatrist in the Psychiatric Institution 'De IJssel'). In: T.I. Oei and M.S. Groenhuijsen (ed.), Forensische Psychiatrie anno 2000 (= Forensic Psy- chiatry anno 2000), Deventer: Gouda Quint 2000, p. 213222

OEI, T.I., GROENHUIJSEN, M.S. (ed.). Forensische Psychiatrie anno 2000, 279-315, Gouda Quint, Deventer 2000.

OEI, T.I. Het middelengebruik in het snelle (weg)verkeer (= Using available means in fastmoving (road)traffic), Verkeersrecht 48, I, 23-26, 2000 (a).

OEI, T.I. Het rapporteren voor de Rechtbank (= Reporting for the Courts), Process, 3/4, 62-63,1999.

OEI, T.I. Detentie(on)geschiktheid bepalen is een justitieel forensisch-psychiatrische taak (= Determining detention (un)suitability is a matter for the judicial forensic psychiatrist). Penose, 14, I, XII-XVII, 2000 (b).

OEI, T.I. Het grote geld lonkt (= Big money attracts), Medisch Contact 55, 45, p. 1587, 2000 (c).

RAES, B.C.M. De tenuitvoerlegging van de maatregel TBS (= The execution of the Court order regulation). In: B.C.M. Raes and F.A.M. Bakker (ed.), De psychiatrie in het Nederlandse recht (= Psychiatry in Dutch low), 133141 Gouda Quint, Deventer 1999.

TUINIER, S. et al., Diagnostiek, neurobiologie en farmacotherapie van agressieve gedragsstoornissen; een overzicht van de stand van zaken (= Diagnostics, neurobiology and pharmacotherapy in respect of agressive behavioural disorders; an overview of the current situation). In: S. Tuinier, W.M.A. Verhoeven, P.J.A. van Panhuis (ed.), Behandelingsstrategieën bij agressieve gedragsstoornissen ( = Treutment strategies for agressive behavioural disorders), 67-101, Bohn Stafleu Van Loghum, Houten/Diegem 2000.

VAN KALMTHOUT, A.M. De medisch-psychiatrische zorg van psychisch gestoorde illegalen: een juridische taxatie (= The medical-psychiatric care of psychically disturbed illegal immigrants: a juridical assessment). In: T.I. Oei, M.S. Groenhuijsen (eds.), Forensische Psychiatrie anno 2000, Deventer: Gouda Quint 2000, p. 223-235. And Also: Top justicie: definitief geen tbs illegalen (= Top justicie: definitely no Court Order illegal immigrants), de Volkskrant (daily national newspaper), 22-11-00, p. 3, in which the Justice Minister is excepted to declare that suspescts are responsible for their actions, when in fact they are not, or whose sense of responsibility is diminished; this then enables the judge to proced with the imposition of a prison sentence, with the result that severely disturbed illegal immigrants ultimately land legally in prison, with the threat hanging over their heads that they could be deported - a situation which is contrary to international agreements. This means that these severely disturbed illegal immigrants are not given adequate tretment, which itself creates furt- 
her difficulties, bearing in mind that their psychic condition requires proper Court Order or General Psychiatric Hospital treatment.

VAN MARLE, H.J.C. Het strafrechtelijk gedragskundig onderzoek (= The criminal behavioural investigation). In: B.C.M. Raes and F.A.M. Bakker eds.) De psychiatrie in het Nederlandse recht (= Psychiatry in Dutch low), 75-94, Gouda Quint, Deventer 1999.

VISSER, W.D. (ed.) Arbeidsongeschiktheid en traumatische (oorlogs)ervaringen (= Unfit to work and traumatic (war)experiences). ICODO Foundation, Utrecht, $2^{\text {nd }}$ edition, p. 19 onwards, 1997.

If a practicing dentist/doctor is found guilty of excessive alchol in-take, he can be forced to end his practice. See Trouw (national daily newspaper), 21-11-00, p. 4, repor- ting the fact that a Groningen dentist had thus been disciplined by his Regional Disciplinary Tribunal.

In the case of agresive or sexual intimidation, whereby transfer and antitransfer symptoms get out of hand. It is abundantly clear that a proper forensic psychotherapy training will not give anyone full immunity to such influences, but a 'vaccination' against it could help.

Dirección para correspondencia:

T.I. Oei

Profesor de Psiquiatría Forense

Faculty of Criminal Law Sciences

Tilburg University

P.O. Box 90153

5000 LE Tilburg

PAÍSES BAJOS 